\title{
VANISHING THETANULLS FOR SOME DIHEDRAL AND CYCLIC COVERINGS OF RIEMANN SURFACES
}

\author{
Robert D. M. Accola
}

\begin{abstract}
Let $W_{g} \rightarrow W_{z}$ be a ramified $p$-sheeted covering of Riemann surfaces of genus $g$ and $z,(z>0)$ where $p$ is an odd prime. Assume that the Galois group is either dihedral or cyclic. Assume, moreover, that the covering is full; that is, there us an integral divisor $E$, of degree $2 r$ on $W_{z}$ which lifts to be canonical on $W_{g}$. Then $g=r p+1$, where $r \geq 1$. Clearly, $W_{g}$ admits $2^{2 z}$ half-canonical linear series of dimension at least $r-z$ arising from divisors on $W_{z}$ whose double is $E$. Theorem 1 Of these $2^{2 z}$ half-canonical linear series $u_{z}\left(=2^{z-1}\left(2^{z}-1\right)\right)$ have dimension at least $r-z+1$. Theorem 2 Let $W_{g}(g=3 r+1, r \geq 3)$ admit four half canonical linear series, three of dimension $r-1$, and one of dimension $r$, whose sum is bi-canonical, where the half-canonical linear series of dimension $r$ is unique. Then $W_{g}$ is a full elliptic-trigonal Riemann surface. (This characterizes the cases $z=1, p=3, g \geq 10$ )
\end{abstract}

\section{Introduction}

Let $\pi_{g z}: W_{g} \rightarrow W_{z}$ be an $m$-sheeted covering of Riemann surfaces of genus $g$ and $z$. In this paper $W_{z}$ will always have positive genus. Then $W_{g}$ has a property not shared by all Riemann surfaces of genus $g$. This special property may be reflected in some special property of the Jacobian of $W_{g}$. We shall be interested in the vanishing properties of the theta function at half-periods (vanishing theta nulls.) By Riemann's solution to the Jacobi inversion problem, this means that we will be interested in the existence of non-generic half-canonical linear series on $W_{g}$. [8] Of course, we do not expect these half-canonical linear series to exist very often, but they do occur in the case $m=2$. [1] We consider the case $m=3$. If $\pi_{g z}$ is unramified then non-generic half-canonical linear series exist, but with ramification one would not expect this to be true in general. The case of interest in this paper is described in the following definition.

Definition. The covering $\pi_{g z}: W_{g} \rightarrow W_{z}$ is said to be full if there exists a linear series $g_{2 r}^{2 r-z}$ on $W_{z}$ which lifts to be canonical on $W_{g}$.

AMS Mathematical Subject Classification. Primary 14H30, Secondary 30F10.

Received February 20, 2004. 
Since two-sheeted coverings are always full, this is a generalization of the case $m=2$ since the existence of non-generic half-canonical linear series on $W_{g}$ is almost always obvious.

If $\pi_{g z}$ is three sheeted and non-cyclic then the Galois group is the dihedral group of order six. It turns out that much of the analysis in the case $m=3$, works equally well in the case of $p$-sheeted dihedral coverings where $p$ is an odd prime.

If $\pi_{g z}$ is full, $r \geq z$, and the lift of $g_{2 r}^{2 r-z}$ is canonical, then $W_{g}$ now admits $2^{2 z}$ half canonical linear series due to the fact that $g_{2 r}^{2 r-z} \equiv 2 g_{r}^{r-z}$ in $2^{2 z}$ different ways. However, in the case of full $p$-sheeted ramified dihedral or cyclic coverings a certain number, $u_{z}$, of these half-canonical linear series have dimension greater than that of $g_{r}^{r-z}$. This is covered in Sections 5 and 6. $\left(u_{z}=2^{z-1}\left(2^{z}-1\right)=\right.$ number of odd theta characteristics in dimension $z$.)

For $p=3, z=1, g \geq 10$, we show when the existence of the four nongeneric half-canonical linear series characterizes full elliptic-trigonal Riemann surfaces. (Section 7) However, we are unable to distinguish between the dihedral and cyclic cases by the methods of this paper.

Section 3 concerns the existence of full ramified $p$-sheeted dihedral coverings and gives a useful characterization. Section 6 concerns the cyclic case. Section 4 gives a very brief account of Weierstrass points for the case $p=3$. (We know of no generalization for $p>3$.) Section 2 on preliminary results is arranged so that the last part of this section is only needed for Section 7.

It is possible for a $W_{10}$ to cover tori in three sheets in four different ways. (For $W_{3 r+1}, r>3$, a three-sheeted covering of a torus is unique.) If those four coverings are full then it can be shown that $W_{10}$ admits an elementary abelian group of order 27. Thus the existence of this group is characterized by certain vanishing properties of the theta function. In this case our inability to distinguished between the cyclic and dihedral cases is overcome by the abundance of coverings. The proof involves an extensive examination of the inequality of Castelnuove-Severi and will be presented in a sequel to this paper.

\section{Definitions, classical theorems, preliminary results}

$W_{g}$ will always stand for a compact Riemann surface of genus $g . K_{g}$ will stand for the canonical linear series $g_{2 g-2}^{g-1}$. The field of meromorphic functions on $W_{g}$ will be denoted $M\left(W_{g}\right)$. If $f \varepsilon M\left(W_{g}\right)$ then $(f)_{a}$ will stand for the a-places of $f$ counted with multiplicity so that the divisor of $f,(f)$, is $(f)_{0}-(f)_{\infty}$. If $D$ is an integral divisor, $|D|$ will stand for the complete linear series of integral divisors linearly equivalent to $D$.

If $\pi: W_{g} \rightarrow W_{z}$ is a $t$-sheeted covering and $D$ is a divisor of degree $d$ on $W_{z}$ then $\pi^{-1}(D)$ will denote the divisor of lifted points with ramification points counted according to multiplicity; consequently deg $\pi^{-1}(D)=\operatorname{td}$. If $P \varepsilon W_{z}$ then $\pi^{-1}(P)$, of degree $t$, will be called a complete fiber of $\pi$. If $D$ is on $W_{g}$ then $\pi(D)$ is the image of $D$ of degree $d$. 
The fibers of a $t$-sheeted covering $W_{g} \rightarrow W_{q}$ will be denoted $\gamma_{t}(q)$, and we will describe the covering by saying that $W_{g}$ admits a $\gamma_{t}(q)$. If $q=0$ then $\gamma_{t}(0)$ is a $g_{t}^{1}$. If $t=2$ then $W_{g}$ is said to be $q$-hyperelliptic $(q=0$, hyperelliptic; $q=1$, elliptic-hyperelliptic.) If $t=3 W_{g}$ is said to be $q$-trigonal $(q=0$, trigonal; $q=1$, elliptic-trigonal) A linear series $g_{n}^{r}$ on $W_{g}$ will be said to be compounded of $\gamma_{t}(q)$ if the divisors of the non-fixed points of $g_{n}^{r}$ are lifted from divisors on $W_{q}$; that is, they are unions of divisors in $\gamma_{t}(q)$.

If $P(X)$ is an irreducible polynomial of degree $n$ in $M\left(W_{z}\right)[X]$ then this polynomial defines an algebraic extension of $M\left(W_{z}\right)$ which lives on a Riemann surface, $W_{g}$, covering $W_{z}$ in $n$ sheets. $M\left(W_{z}\right)$ is isomorphic to a subfield of $M\left(W_{g}\right)$ of index $n$. In this context $M\left(W_{z}\right)$ will stand for the field on $W_{z}$ or for its isomorphic image on $W_{g}$. No confusion should result. If $P(X)=X^{n}-f$ then we will say that $f^{(1 / n)}$ defines the cyclic covering $W_{g} \rightarrow W_{z}$.

With one exception (Theorem 6.1) $p$ will always stand for an odd prime. A $p$-sheeted dihedral covering $\pi_{g z}: W_{g} \rightarrow W_{z}$ will have two types of ramification points. Those of multiplicity $p$ will be called total. Those of multiplicity 2 will be called ordinary. The complete fibers of $\pi_{g z}$ are of three types: a single total ramification point, $(p-1) / 2$ ordinary ramifications points together with a single unramified point, or $p$ unramified points. Suppose $\pi_{g z}$ has $s$ total ramification points and ordinary ramification points over $n$ points of $W_{z}$. Then the total ramification of the covering is $(p-1) s+((p-1) / 2) n$. The Riemann-Hurwitz formula gives

$$
2 g-2=p(2 z-2)+((p-1) / 2)(2 s+n)
$$

where $n$ is always even. If the covering is full then $2 s+n \equiv 0(\bmod 2 p)$ since $p$ divides $2 g-2$.

Let $W_{a}$ be a Riemann surface of genus $a$ admitting a group of automorphisms, $G$, isomorphic to the dihedral group of order $2 p$. We will always write $G=\langle\psi, \varphi\rangle$ where $\Psi^{p}=\varphi^{2}=e$. Let $W_{g}=W_{a} /\langle\varphi\rangle, \quad W_{h}=W_{a} /\langle\psi\rangle$ and $W_{z}=W_{a} / G$. Then $a+2 z=2 g+h . \quad$ [7]

THEOREM 2.1 [7]. Let $g_{n}^{r}$ be a complete linear series on $W_{z}$. Let $g_{2 n}^{r+c}$ (resp $\left.g_{p n}^{r+b}\right)$ be the completion of the lift of $g_{n}^{r}$ to $W_{h}\left(\right.$ resp $\left.W_{g}\right)$. Then the completion of $g_{n}^{r}$ lifted to $W_{a}$ is $g_{2 p n}^{r+c+2 b}$.

In the theorem let $D_{z}$ be a divisor in $g_{n}^{r}$ on $W_{z}$. On $W_{a}$ let $D_{a}$ be the divisor of degree $2 p n$ which is the lift of $D_{z}$. The vector space of meromorphic functions on $W_{a}$ which are multiples of $D_{a}$ is a complex represention of $G$ of dimension $r+c+2 b+1$.

COROLlaRY 2.2. The multiplicity of the trivial representation is $r+1$. The multiplicity of the non-trivial one dimentional representation is $c$. The sum of the multiplicities of the irreducible representations of dimension two is $b$.

We call attention to an important obvious fact. 
COROLlaRY 2.3. If there is a divisor in $g_{2 p n}^{r+c+2 b}$ which is not invariant under $\langle\psi\rangle$ then $b$ is positive.

We now quote some classical theorems with abbreviations for later reference.

(RR) Riemann-Roch If $g_{n}^{r}$ is complete then $r=n-g+i$ where $i$ is the index of specialty.

(BN) Brill-Noether If $g_{n}^{r}$ and $h_{m}^{s}$ are complete and their sum is $K_{g}$, then $n-2 r=m-2 s$.

(Cliff) Clifford's theorem If $g_{n}^{r}$ is special then $n-2 r \geq 0$. Equality in nontrivial cases implies $W_{g}$ is hyperelliptic.

(CS) Castelnuovo-Severi inequality If $W_{g}$ admits a $\gamma_{m}(s)$ and a $\gamma_{n}(t)$ and the two coverings admit no non-trivial common factorizations then

$$
g \leq m s+n t+(m-1)(n-1)
$$

and equality has further consequences.

We now state a series of results with either proofs, references, or whose proofs follow from standard techniques.

Lemma 2.4. Let $\pi: W_{g} \rightarrow W_{z}$ be a p-sheeted covering with positive ramification. Suppose $A$ and $B$ are integral divisors on $W_{z}$ so that $\pi^{-1}(A) \equiv \pi^{-1}(B)$. Then $A \equiv B$.

Lemma 2.5. Let $A$ and $B$ be integral divisors on $W_{g}$ so that $2 A \equiv p B$ where $\operatorname{deg} B \geq 2 g$. Then there exists an integral divisor $C$ so that $B=2 C$ and $A=p C$.

The material in this section from now on will be needed only in Section 7 .

Lemma 2.6. Let $g_{n}^{r}$ and $h_{n}^{s}$ be two different linear series so that $2 g_{n}^{r} \equiv 2 h_{n}^{s}$. Then on an unramified two-sheeted covering, $W_{2 g-1}$, of $W_{g}$ there is a linear series $g_{2 n}^{r+s+1}$ which is the completion of the lift of $g_{n}^{r}\left(\right.$ and $\left.h_{n}^{s}\right)$. If one of the two linear series is simple then so also is $g_{2 n}^{r+s+1}$.

Proof. Let $D$ and $E$ be divisors in $g_{n}^{r}$ and $h_{n}^{s}$ respectively. Let $f$ be a function whose divisor is $2 D-2 E$. Then $W_{2 g-1}$ is defined by $\sqrt{f}$. If $D^{\prime}$ is the lift of $D$ then the dimension of the multiples of $D^{\prime}$ is $(r+1)+(s+1)$. If $g_{n}^{r}$ is simple there are more multiples of $D^{\prime}$ than there are of $D$. So $g^{r+s+1}$ is simple.

Lemma 2.7. Let $W_{g}$ admit 4 distinct linear series, $g_{n}^{a}, h_{n}^{b}, k_{n}^{c}$, and $l_{n}^{d}$ so that

i) $2 g_{n}^{a} \equiv 2 h_{n}^{b} \equiv 2 k_{n}^{c} \equiv 2 l_{n}^{d}$

ii) $g_{n}^{a}+h_{n}^{b} \equiv k_{n}^{c}+l_{n}^{d}$

Then there exists an unramified 4-sheeted Galois covering $W_{4 g-3} \rightarrow W_{g}$ (the Galois group is the four group) and on $W_{4 g-3}$ there exists a $g^{a+b+c+d+3}$ which is the completion of the lift of $g_{n}^{a}$. If one of the four linear series on $W_{g}$ is simple so also is $g^{a+b+c+d+3}$. 
Proof. Apply the previous lemma twice.

Lemma 2.8. Suppose $W_{h}$ admits a $\gamma_{2}(g), g \geq 6$, and is also elliptic-trigonal. Then $W_{g}$ is elliptic-trigonal or trigonal.

Proof. Since $h \geq 11, W_{h}$ admits a unique $\gamma_{3}(1)$ (CS). The involution whose quotient is $W_{g}$ must permute the divisors of $\gamma_{3}(1)$. Thus $W_{g}$ admits a $\gamma_{3}(q)$ where $q=1$ or 0 .

A linear series $g_{m}^{s}$ will be said to impose $t$ (linear) conditions on a complete $g_{n}^{r}$ if $\left|g_{n}^{r}-g_{m}^{s}\right|=g_{n-m}^{r-t}$.

Lemma 2.9 [3]. Let $g_{g-1}^{r}$ be half canonical. Let $g_{m}^{1}$ be a linear series without fixed points where $m \leq 2 r+1$. Then $g_{m}^{1}$ imposes at most $[m / 2]$ conditions on $g_{g-1}^{r}$.

Lemma 2.10 [3]. Let $g_{m}^{s}(s \geq 2)$ be a simple linear series without fixed points with $m-s \leq 2 r$. Then $g_{m}^{s}$ imposes at most $[(m-s+1) / 2]$ conditions on any halfcanonical $g_{g-1}^{r}$. Thus such a half-canonical $g_{g-1}^{r}$ must be simple.

Lemma 2.11 [3]. Suppose $W_{g}$ admits a $\gamma_{2}(q)$. Then every half-canonical $g_{g-1}^{q+1}$ is compounded of $\gamma_{2}(q)$. (Thus $g_{g-1}^{r}$ being simple and half-canonical implies that $r \leq q$.)

Lemma $2.12\left([1]\right.$, p 51). Suppose $W_{g}$ admits a $\gamma_{2}(q)$ and $r=[(g-1) / 2]-$ $q \geq 0$. Then $W_{g}$ admits many half-canonical $g_{g-1}^{r}$ 's. If $g$ is odd the number is at least $4^{q}$. If $g$ is even the number is at least $(g+2-2 q) 4^{q}$.

Lemma 2.13. Suppose $W_{g}$ admits a $\gamma_{3}(q)$. Let $D$ be an integral divisor of degree 3 so that $|2 D|$ is compounded of $\gamma_{3}(q)$. Then $D$ is in $\gamma_{3}(q)$.

Proof. Let $D=x+y+z . \quad 2 x+2 y+2 z$ is a union of 2 divisors in $\gamma_{3}(q)$. If $D$ is not in $\gamma_{3}(q)$, say $2 x+y$ is in $\gamma_{3}(q)$. But a divisor in $\gamma_{3}(q)$ is determined by any point in it, so $2 x+y=y+2 z$ or $x=z$. $D$ is in $\gamma_{3}(q)$ after all.

Lemma 2.14 [3]. Suppose $W_{g}$ admits a $g_{4}^{1}$. Let $D$ be an integral divisor of degree 4 not in $g_{4}^{1}$, so that $|2 D|=2 g_{4}^{1}=g_{8}^{2}$. Then there exist two disjoint integral divisors of degree $2, P$ and $Q$, so that $D=P+Q$ and $|2 P|=|2 Q|=g_{4}^{1}$.

(The proof uses the same kind of reasoning as in Lemma 2.13.)

Lemma 2.15. Suppose $W_{3 r+1}(r \geq 2)$ is trigonal and admits a half-canonical $g_{3 r}^{r}$ (necessarily compounded of $g_{3}^{1}$ by Lemma 2.9.) Then $W_{3 r+1}$ does not admit a complete half-canonical $g_{3 r}^{r-1}$. 
Proof. Suppose the contrary. $g_{3 r}^{r-1}$ is compounded of $g_{3}^{1}$ so that $g_{3 r}^{r-1}=$ $(r-1) g_{3}^{1}+D$ where $D$ is not in $g_{3}^{1}$. Since $K_{3 r+1} \equiv(2 r) g_{3}^{1}$ we see that $2 D \equiv 2 g_{3}^{1}$. Since $3 r+1 \geq 7,\left|2 g_{3}^{1}\right|=g_{6}^{2}$. Lemma 2.13 gives the contradiction.

Lemma 2.16 (Castelnuovo, [2]). Let $g_{n}^{r}$ be a simple linear series on $W_{g}$. $(n>2 r)$ Then $2 g_{n}^{r}$ has dimension $3 r-1+\varepsilon, \varepsilon \geq 0$.

Lemma 2.17. Suppose $W_{3 r+1}$ admits a simple half-canonical $g_{3 r}^{r}$ and a $g_{m}^{1}$ which imposes two conditions on $g_{3 r}^{r}$. If $r>5$ then $m=4$.

Proof. Since $g_{3 r}^{r}$ is simple $m \geq 4$. Let $D$ be an integral divisor of $r-2$ points in general position on $W_{3 r+1}$. Then $g_{3 r}^{r}-D\left(=g_{2 r+2}^{2}\right)$ gives a plane model for $W_{3 r+1}$ with $r-2$ singularities of degree $m-1$ and one of multiplicity $2 r+2-m$ and possibly other singularities. Then

$$
3 r+1 \leq[(2 r+1)(2 r)-(r-2)(m-1)(m-2)-(2 r+2-m)(2 r+1-m)] / 2
$$

which simplifies to: $m \leq 4 r /(r-1)$. Since $r>5$ we have $m \leq 4$.

LemMa 2.18 [2]. Let $g_{n}^{r}$ and $g_{m}^{s}$ be two distinct linear series where $g_{n}^{r}$ is simple and $r \geq s$. Then $g_{n}^{r}+g_{m}^{s}$ has dimension at least $r+2 s$.

\section{Existence}

Any ramified dihedral covering of odd prime order $p, \pi_{g z}: W_{g} \rightarrow W_{z}$, arises in accordance with the following procedure. There is given a meromorphic function $y: W_{z} \rightarrow \mathbf{P}^{1} . \quad \sqrt{y}(:=Y)$ defines a two-sheeted covering $\pi_{h z}: W_{h} \rightarrow W_{z}$, ramified over the zeros and poles of $y$ whose orders are odd. Let $Z=(1-Y) /$ $(1+Y)$. Then a $p$-sheeted cyclic covering $\pi_{a h}: W_{a} \rightarrow W_{h}$ is defined by $Z^{(1 / p)}$ $(:=U)$ and is ramified over those zeros and poles of $Z$ whose orders are not divisible by $p$. The map $\pi_{a z}:=\pi_{h z} \circ \pi_{a h}$ is a Galois covering with Galois group, $G$, isomorphic to the dihedral group of order $2 p . \quad G=\langle\varphi, \psi\rangle$ where $\varphi^{*} U=U^{-1}$ and $\psi^{*} U=\omega U, \omega^{p}=1$, and $\omega \neq 1$. Then $W_{g}=W_{a} /\langle\varphi\rangle$ and the covering map $\pi_{a g}: W_{a} \rightarrow W_{g}$ is the Galois closure of the $p$-sheeted dihedral covering $\pi_{g z}: W_{g} \rightarrow W_{z}$. We will prove the assertions in this paragraph only in the following special situation.

THEOREM 3.1. A ramified p-sheeted dihedral covering is full if and only if it arises by the above procedure with a y satisfying the following:

$$
\begin{aligned}
& (y)_{0}=B_{z n} \text {, a divisor of } n \text { distinct points, } n \text { even } \\
& (y)_{\infty}=2 C_{z \mu}, C_{z \mu} \text { a divisor of degree } \mu(=n / 2) \\
& (y)_{1}=(p-2) D_{z s}+p D_{z t}, \quad D_{z s} \text { a divisor of } s \text { distinct points, } \operatorname{deg} D_{z t}=t \\
& (\operatorname{deg} y=n=2 \mu=(p-2) s+p t)
\end{aligned}
$$


Proof. Assuming the existence of such a $y$, we follow the above procedure keeping track of significant divisors.

Let $\sqrt{y}(:=Y)$ define $\pi_{h z}: W_{h} \rightarrow W_{z} . \quad$ Let $Z=(1-Y) /(1+Y) . \quad Y$ and $Z$ are in $M\left(W_{h}\right)$, and both are of degree $n$.

$(Z)_{1}=(Y)_{0}=B_{h n}$, the $n$ ramified points of $\pi_{h z}$

$(Z)_{-1}=(Y)_{\infty}=C_{h \mu}, \mu$ complete unramified fibers of $\pi_{h z} ; \operatorname{deg} C_{h \mu}=2 \mu=n$

$(Z)_{0}=(Y)_{1}=(p-2) D_{h s}+p D_{h t}$ where $D_{h s}$ and $D_{h t}$ lie above $D_{z s}$ and $D_{z t}$

$(Z)_{\infty}=(Y)_{-1}=(p-2) E_{h s}+p E_{h t}$ where $E_{h s}$ and $E_{h t}$ lie above $D_{z s}$ and $D_{z t}$

Now $Z^{(1 / p)}(:=U)$ defines $\pi_{a h}: W_{a} \rightarrow W_{h}$, a $p$-sheeted cyclic covering. $W_{h}=W_{a} /\langle\psi\rangle$ where $\psi^{*} U=\omega U$, as before.

$(U) \omega^{i}=B_{a n}^{i}$ lying above $B_{h n}, \quad i=0,1,2, \ldots, p-1 . \operatorname{deg} B_{a n}^{i}=n$.

$(U)-\omega^{i}=C_{a \mu}^{i}$ lying above $C_{h \mu}, \quad i=0,1,2, \ldots, p-1 . \operatorname{deg} C_{a \mu}^{i}=n$.

$(U)_{0}=(p-2) D_{a s}+D_{a t}$

$(U)_{\infty}=(p-2) E_{a s}+E_{a t}$

where $D_{a s}+E_{a s}$ are the ramified points of $\pi_{a h}, D_{a t}$ and $E_{a t}$ are each composed of $t$ complete unramified fibers of $\pi_{a h}$, and $D_{a t}+E_{a t}$ is composed of $t$ complete fibers of $\pi_{a z}$.

We may assume that the fixed points of $\varphi$ are $B_{a n}^{0}$. Now

$$
K_{a} \equiv \pi_{a z}^{-1}\left(K_{z}\right)+\sum_{i=0}^{p-1} B_{a n}^{i}+(p-1)\left(D_{a s}+E_{a s}\right)
$$

By the linear equivalence of divisors where $U$ takes different values

$$
\begin{aligned}
\sum_{i=1}^{p-1} B_{a n}^{i} & \equiv((p-1) / 2)\left[(p-2)\left(D_{a s}+E_{a s}\right)+\left(D_{a t}+E_{a t}\right)\right] . \quad \text { Consequently } \\
K_{a} & \equiv \pi_{a z}^{-1}\left(K_{z}\right)+B_{a n}^{0}+((p-1) / 2)\left[p\left(D_{a s}+E_{a s}\right)+\left(D_{a t}+E_{a t}\right)\right] \\
& \equiv B_{a n}^{0}+\pi_{a z}^{-1}\left(K_{z}+((p-1) / 2)\left[D_{z s}+D_{z t}\right]\right) . \quad \text { But } \\
K_{a} & \equiv B_{a n}^{0}+\pi_{a g}^{-1}\left(K_{g}\right), \quad \text { so by Lemma } 2.4 \\
K_{g} & \equiv \pi_{g z}^{-1}\left(K_{z}+((p-1) / 2)\left[D_{z s}+D_{z t}\right]\right) .
\end{aligned}
$$

Thus $\pi_{g z}: W_{g} \rightarrow W_{z}$ is a full $p$-sheeted dihedral covering.

Now we assume for a ramified $p$-sheeted dihedral covering $\pi_{g z}: W_{g} \rightarrow W_{z}$ that $K_{g} \equiv \pi_{g z}^{-1}\left(G_{0}\right)$ for a fixed divisor $G_{0}$ on $W_{z}$. (By Lemma 2.4 any two such $G_{0}$ 's are linearly equivalent.) We must show that this covering arises from a 
meromorphic function $y$ on $W_{z}$ as described in the statement of the theorem. We first show that there are, in deed, ordinary ramification points.

Lemma 3.2. Let $\pi_{g z}: W_{g} \rightarrow W_{z}$ be a ramified p-sheeted full covering with only total ramification points. Then the covering is cyclic.

Proof of Lemma 3.2. Let $B$ be the divisor of ramification points of $\pi_{g z}$. Then

$$
\begin{aligned}
& K_{g} \equiv(p-1) B+\pi_{g z}^{-1}\left(K_{z}\right) . \quad \text { But } \\
& K_{g} \equiv \pi_{g z}^{-1}\left(G_{0}\right)
\end{aligned}
$$

for a divisor $G_{0}$ on $W_{z}$. The right hand sides of these last two equivalences, being linearly equivalent, are the zeros and poles of a function in $M\left(W_{g}\right)$ whose $p$ th power is a function in $M\left(W_{z}\right)$. Thus the covering is cyclic.

Our covering $\pi_{g z}$ is assured of ordinary ramification points. Let $\pi_{a g}$ : $W_{a} \rightarrow W_{g}$ be the Galois closure of $\pi_{g z}$. Let the Galois group be $G=\langle\varphi, \psi\rangle$, $W_{g}=W_{a} /\langle\varphi\rangle$ and $W_{h}=W_{a} /\langle\psi\rangle$. Let $B_{a n}^{0}$ be the ramification divisor of $\pi_{a g}$, a divisor of even degree $n$ on $W_{a}$. Then

$$
K_{a} \equiv B_{a n}^{0}+\pi_{a g}^{-1}\left(K_{g}\right)
$$

Letting $K_{a o}$ be a divisor in $K_{a}$ invariant under $G$ we have

$$
K_{a o} \equiv B_{a n}^{0}+\pi_{a z}^{-1}\left(G_{0}\right) .
$$

Let $L=\left\{f \varepsilon M\left(W_{a}\right) \mid(f) \geq \pi_{a z}^{-1}\left(G_{0}\right)-K_{a o}\right\}$.

$L$ is a complex representation for $G$. It contains a function (whose zeros and poles are the two sides of (3.2)) with a divisor not invariant under $\psi$. Thus it must contain an irreducible two dimensional representation $\left\langle f_{1}, f_{2}\right\rangle$, where we may assume $\left(\tau^{p}=1, \tau \neq 1\right)$

$$
\psi^{*} f_{1}=\tau f_{1}, \quad \psi^{*} f_{2}=\tau^{-1} f_{2}, \quad \varphi^{*} f_{1}=f_{2}, \quad \varphi^{*} f_{2}=f_{1} .
$$

Let $U=f_{1} / f_{2}$ and let $V=(1-U) /(1+U)$. Then $\varphi^{*} V=-V$ and so $V$ defines $\pi_{a g}$.

Now assume that the degree of $U$ (and therefore $V$ ) is $n$.

We assert that $B_{a n}^{0}=(V)_{0}$ or $(V)_{\infty}$. Observe that $B_{a n}^{0} \equiv(V)_{0}$. To prove the assertion let $X$ be a function on $W_{a}$ where $(X)=B_{a n}^{0}-(V)_{0}$. Since the zeros and poles of $X$ are invariant under $\varphi, \varphi^{*} X=\lambda X$ where $\lambda=1$ or -1 . Assume $\lambda=1$. Then $X$ is invariant under $\varphi$, and $X$ has $B_{a n}^{0}$ for zeros, a divisor which contains no complete fiber of $\pi_{a g}$. Therefore $X$ is constant and $B_{a n}^{0}=(V)_{0}$. (If $\lambda=-1$ replace $X$ with $V X$ and deduce that $B_{a n}^{0}=(V)_{\infty}$.)

We now assume that $B_{a n}^{0}=(V)_{0}$. Since $U=(1-V) /(1+V)$ we have $(U)_{1}=B_{a n}^{0}$. Let $\omega=\tau^{2}$. Then $\psi^{*} U=\omega U$. It follows that $(U)_{\omega} i=\psi^{i}\left(B_{a n}^{0}\right)$ $\left(:=B_{a n}^{i}\right) \quad i=0,1, \ldots, p-1$ give the $p$ divisors, of degree $n$, of the ordinary ramification points of $\pi_{a z}$. 
Now $\psi^{*} U^{p}=U^{p}$ and so $U$ defines $\pi_{a h}$, and the ramification points of $\pi_{a h}$ are the zeros and poles of $U$ whose orders are not divisible by $p$. Let $Z=U^{p}$, a function on $W_{h}$. Let

$$
(Z)_{0}=D_{h 1}+2 D_{h 2}+\cdots+(p-1) D_{h(p-1)}+p D_{h p}
$$

where the $D_{h i}, i=1,2, \ldots p-1$ (but not necessarily $p$ ) are pairwise coprime divisors of points counted with multiplicity one. On $W_{a}$

$$
(U)_{0}=D_{a 1}+2 D_{a 2}+\cdots+(p-1) D_{a(p-1)}+D_{a p}
$$

where the $D_{a i}, i=1,2, \ldots(p-1)$ are ramification points of $\pi_{a h}$ lying above the $D_{h i}$, and $D_{a p}$ is a set of $\left(\operatorname{deg} D_{h p}\right)$ complete fibers of $\pi_{a h}$.

If $\Phi$ is the involution of $W_{h}$ which lifts to $\varphi$ and if

$$
(Z)_{\infty}=E_{h 1}+2 E_{h 2}+\cdots+(p-1) E_{h(p-1)}+p E_{h p}
$$

then $\Phi$ interchanges the $D_{h i}$ 's and the $E_{h i}$ 's and

$$
(U)_{\infty}=E_{a 1}+2 E_{a 2}+\cdots+(p-1) E_{a(p-1)}+E_{a p}
$$

with definitions analogous to those of the $D_{a i}$ 's.

We now show that $D_{h i}=0$ for $i \neq p-2, p$. Then setting $Y=(1-Z) /$ $(1+Z)$, noting that $\Phi^{*} Y=-Y$, and setting $y=Y^{2}$, the proof will be complete for $\operatorname{deg} U=n$. (If in the assertion above, $B_{a n}^{0}=(V)_{\infty}$ then set $y=Y^{-2}$.)

On $W_{a}$ again note that $(U)_{0} \equiv(U)_{\infty} \equiv(U)_{1} \equiv B_{a n}^{i}$ for all $i$.

$$
\begin{aligned}
K_{a} \equiv & \sum_{i=0}^{p-1} B_{a n}^{i}+\sum_{j=1}^{p-1}(p-1)\left(D_{a j}+E_{a j}\right)+\pi_{a z}^{-1}\left(K_{z}\right) \\
\sum_{i=0}^{p-1} B_{a n}^{i} \equiv & B_{a n}^{0}+((p-1) / 2)\left(\sum_{j=1}^{p-1} j\left(D_{a j}+E_{a j}\right)+\left(D_{a p}+E_{a p}\right)\right) \quad \text { Therefore } \\
K_{a} \equiv & B_{a n}^{0}+\pi_{a z}^{-1}\left(K_{z}\right)+((p-1) / 2)\left(D_{a p}+E_{a p}\right) \\
& +\sum[((p-1) j) / 2+(p-1)]\left(D_{a j}+E_{a j}\right)
\end{aligned}
$$

From the last sum extract the $j=p-2$ term and denote what is left by a primed summation.

$$
\begin{aligned}
K_{a} \equiv & B_{a n}^{0}+\pi_{a z}^{-1}\left(K_{z}\right)+((p-1) / 2)\left(D_{a p}+E_{a p}\right)+((p-1) / 2) p\left(D_{a(p-2)}\right. \\
& \left.+E_{a(p-2)}\right)+\sum^{\prime}[]\left(D_{a j}+E_{a j}\right)
\end{aligned}
$$

where none of the integers in the brackets are divisible by $p$. Note that the second, third, and fourth terms denote sets of complete fibers of $\pi_{a z}$, namely

$$
\pi_{a z}^{-1}\left(K_{z}+((p-1) / 2)\left(D_{z p}+D_{z(p-2)}\right)\right)
$$

for suitable divisors $D_{z p}$ and $D_{z(p-2)}$ on $W_{z}$ where $\pi_{a z}$ has total ramification points above $D_{z(p-2)}$ and is unramified above $D_{z p}$. Since 


$$
K_{a} \equiv B_{a n}^{0}+\pi_{a g}^{-1}\left(K_{g}\right) \equiv B_{a n}^{0}+\pi_{a z}^{-1}\left(G_{0}\right) \text {, }
$$

descending to $W_{g}$ by Lemma 2.4 we have

$$
\pi_{g z}^{-1}\left(G_{0}\right) \equiv \pi_{g z}^{-1}\left(K_{z}+((p-1) / 2)\left(D_{z p}+D_{z(p-2)}\right)\right)+\sum^{\prime}[] D_{g j}
$$

for suitable divisors $D_{g j}$ on $W_{g}$. The $D_{g j}(j<p)$ are the total ramification points for $\pi_{g z}$. If $\sum^{\prime}[] D_{g z}$ is not zero in the last equivalence then the right and left hand sides are the zeros and poles of a function on $W_{g}$ whose $p$ th power is a function in $M\left(W_{z}\right)$. This implies that $\pi_{g z}$ is a cyclic covering. This contradiction proves the theorem in case the degree of $U$ is $n$.

Now assume that the degree of $U$ is less than $n$. Then

$$
\begin{aligned}
& \left(f_{1}\right)=A_{1}+F+\pi_{a z}^{-1}\left(G_{0}\right)-K_{a o} . \\
& \left(f_{2}\right)=A_{2}+F+\pi_{a z}^{-1}\left(G_{0}\right)-K_{a o}
\end{aligned}
$$

where $\left(A_{1}, A_{2}\right)=0, F \neq 0$, and $F$ is invariant under $\varphi$. Again let $U=f_{1} / f_{2}$, $V=(1-U) /(1+U), \varphi^{*} V=-V$ and $V$ defines $\pi_{a g}$. Define $X$ by $(X)=B_{a n}^{0}-$ $\left[(V)_{0}+F\right]$. Then $\varphi^{*} X=\lambda X$, and $\lambda=1$ or -1 . If $\lambda=1$, then $X$ is invariant under $\varphi$ and $B_{a n}^{0}=(V)_{0}+F$. If $\lambda=-1$, then $V X$ is invariant under $\varphi$ and $B_{a n}^{0}=(V)_{\infty}+F$. Thus $F$ consists of part of $B_{a n}^{0}$.

We wish to show that $\operatorname{Deg} F=n / 2$.

Assume $\lambda=1$. (The case $\lambda=-1$ is handled in an analogous way.) $(U)_{1}=(V)_{0}$ and so $B_{a n}^{0} \equiv(U)_{1}+F$. Define $B_{a n}^{1}:=\psi\left(B_{a n}^{0}\right)$. Then $B_{a n}^{1}=$ $\psi\left((U)_{1}\right)+\psi(F)$ and $(U)_{\omega}=\psi\left((U)_{1}\right)$. Consequently $(U)_{1}+F \equiv(U)_{\omega}+\psi(F)$. Thus we have two functions, $\theta_{1}, \theta_{2}$ with divisors:

$$
\left(\theta_{1}\right)=(U)_{1}-(U)_{\omega}, \quad\left(\theta_{2}\right)=F-\psi(F)
$$

and $B_{a n}^{0}=(U)_{1}+F$, the ramification divisor of $\varphi$. Apply CS to the two covers $\theta_{1}: W_{a} \rightarrow \mathbf{P}^{1}$ and $\pi_{a g}: W_{a} \rightarrow W_{g}$. Since $\theta_{1}$ cannot be in $M\left(W_{g}\right)$ we have $a \leq\left(\operatorname{deg} \theta_{1}-1\right)+2 g$. But $a=2 g-1+n / 2$. Consequently $\operatorname{deg} \theta_{1} \geq n / 2$. Similarly $\operatorname{deg} \theta_{2} \geq n / 2$. Since $\operatorname{deg} \theta_{1}+\operatorname{deg} \theta_{2}=n$ we conclude that $\operatorname{deg} F=$ $\operatorname{deg} A_{1}=\operatorname{deg} A_{2}=n / 2$.

Now $B_{a n}^{0}$ is part of $(V)_{0}+(V)_{\infty}$ since $V$ defines $\pi_{a g}$. But $\operatorname{deg} F=$ $\operatorname{deg} U=\operatorname{deg} V=n / 2 ; \quad$ therefore $\quad B_{a n}^{0}=(V)_{0}+(V)_{\infty}=(U)_{1}+(U)_{-1}=\left(U^{2}\right)_{1}$, and $\psi^{*} U^{2}=\omega^{2} U^{2}$.

Thus $U^{2}$ satisfies the properties of $U$ (when $\operatorname{deg} U=n$ ), and we may proceed with the rest of the proof as before to obtain our desired $y$. Similarly for the case $B_{a n}^{0}=(V)_{\infty}+F$. This completes the proof of the theorem.

Note that in the first part of the theorem where we proceeded from $y$ to the full covering $\pi_{g z}$, we can achieve an irreducible two dimensional representation in $L$ by defining $f_{1}$ and $f_{2}$ as follows:

$$
\begin{aligned}
& \left(f_{1}\right)=(p-2) D_{a s}+D_{a p}+\pi_{a z}^{-1}\left(G_{0}\right)-K_{a o} \\
& \left(f_{2}\right)=(p-2) E_{a s}+E_{a p}+\pi_{a z}^{-1}\left(G_{0}\right)-K_{a o}
\end{aligned}
$$


Thus we see that for a given full covering $\pi_{a g}$ there are at most $(\operatorname{dim} L) / 2$ possible $y$ 's which give rise to it according to the procedure at the beginning of this section. This allows us to compute, naively, the dimension, $\alpha$, in moduli space of such $W_{g}$ 's.

By the Riemann Hurwitz formula for $y: W_{z} \rightarrow \mathbf{P}^{1}$ we have

$$
2 z-2=-2 n+n / 2+(p-3) s+(p-1) t+\alpha
$$

Since $n=(p-2) s+p t$ we have $\alpha=2 z-2+(n / 2)+(n+2 s) / p$.

\section{Weierstrass points $(p=3)$}

We draw attention to the fact that for full three-sheeted dihedral coverings (with $g$ sufficiently large) ordinary ramification points are Weierstrass points.

THEOREM 3. If $W_{g} \rightarrow W_{z}$ is a full three-sheeted covering and $g \geq 4+6 z$, then each ordinary ramification point of the covering is a Weierstrass point.

Proof. Suppose $g_{(2 g-2) / 3}^{x}$ on $W_{z}$ lifts to be canonical on $W_{g}$. If $P$ is an ordinary ramification point then $x(2 P)$ will be special if $2 x \geq g$. Now $x \geq((2 g-2) / 3)-z$, so a sufficient condition that $P$ be a Weierstrass point is $2(((2 g-2) / 3)-z) \geq g$.

\section{Half-canonical linear series on dihedral coverings}

Assuming a full ramified $p$-sheeted dihedral covering $\pi_{g z}$, there is a linear series $g_{2 r}^{2 r-z}$ on $W_{z}$ which lifts to be canonical on $W_{g}$, and so $2 g-2=2 r p$. Since

$$
2 g-2=p(2 z-2)+((p-1) / 2)(2 s+n)
$$

and $2 p$ divides $2 s+n$, we see that

$$
2 r \geq(2 z-2)+(p-1) \geq 2 z
$$

since $p \geq 3$. Thus $g_{2 r}^{2 r-z}$ is not special and is complete. Consequently $g_{2 r}^{2 r-z} \equiv$ $2 g_{r}^{r-z}$ for $2^{2 z}$ linear series $g_{r}^{r-z}$ (which need not be complete), and so $W_{g}$ admits $2^{2 z}$ half-canonical linear series of dimension at least that of $g_{r}^{r-z}$.

THeOREM 5.1. Of those $2^{2 z}$ half-canonical linear series $u_{z}\left(=2^{z-1}\left(2^{z}-1\right)\right)$ have dimension greater than that of the corresponding $g_{r}^{r-z}$.

Proof. Continue the notation of the previous sections.

$$
\begin{aligned}
& K_{a} \equiv B_{a n}^{0}+\pi_{a g}^{-1}\left(K_{g}\right) \equiv B_{a n}^{0}+\pi_{a z}^{-1}\left(g_{2 r}^{2 r-z}\right) \\
& K_{g} \equiv \pi_{g z}^{-1}\left(g_{2 r}^{2 r-z}\right) \equiv \pi_{g z}^{-1}\left(K_{z}\right)+B_{g n}+(p-1) D_{g s}
\end{aligned}
$$

where $D_{g s}$ is the set of $s$ total ramification points of $\pi_{g z}$, and $B_{g n}$ is the set of 
$n(p-1) / 2$ ordinary ramification points which lie in $n$ stacks of $(p-1) / 2$ points over the $n$ points of $B_{z n}$ on $W_{z}$. Map the elements represented by (4.2) back onto $W_{z}$ by $\pi_{g z}$.

$$
p q_{2 r}^{2 r-z} \equiv p K_{z}+((p-1) / 2) B_{z n}+(p-1) D_{z s} .
$$

Now two-sheeted coverings are always full (Theorem 6.1), so there is a linear series, $f$, of degree $2 z-2+n / 2$ on $W_{z}$ so that

$$
K_{h} \equiv \pi_{h z}^{-1}\left(K_{z}\right)+B_{h n} \equiv \pi_{h z}^{-1}(f)
$$

Mapping onto $W_{z}$ by $\pi_{h z}$ gives

$$
2 f \equiv 2 K_{z}+B_{z n}
$$

Rewriting (4.3):

$$
p g_{2 r}^{2 r-z} \equiv K_{z}+((p-1) / 2)\left(2 K_{z}+B_{z n}+2 D_{z s}\right),
$$

and so

$$
p g_{2 r}^{2 r-z} \equiv K_{z}+((p-1) / 2)\left(2 f+2 D_{z s}\right)
$$

There are, generically, $u_{z}$ half-canonical $g_{z-1}^{0}$ 's on $W_{z}$ so that for any such $g_{z-1}^{0}$ the last equivalence can be written

$$
p g_{2 r}^{2 r-z} \equiv 2\left[g_{z-1}^{0}+((p-1) / 2)\left(f+D_{z s}\right)\right]
$$

By Lemma 2.5 there is a $g_{r}^{r-z}$ so that

$$
g_{2 r}^{2 r-z} \equiv 2 g_{r}^{r-z} \quad \text { and } \quad g_{z-1}^{0}+((p-1) / 2)\left(f+D_{z s}\right) \equiv p g_{r}^{r-z}
$$

Considering $\pi_{a h}$ we see that

$$
\begin{aligned}
& K_{a} \equiv \pi_{a h}^{-1}\left(K_{h}\right)+(p-1)\left(D_{a s}+E_{a s}\right) \quad \text { or } \\
& K_{a} \equiv \pi_{a z}^{-1}(f)+(p-1)\left(D_{a s}+E_{a s}\right)
\end{aligned}
$$

Combining this with (4.1) we have

$$
\pi_{a z}^{-1}(f)+(p-1)\left(D_{a s}+E_{a s}\right) \equiv \pi_{a z}^{-1}\left(2 g_{r}^{r-z}\right)+B_{a n}^{0}
$$

Multiply (4.7) by $(p-1) / 2$, and substitute (4.5) eliminating $\pi_{a z}^{-1}(((p-1) / 2) f)$

$$
\begin{gathered}
\pi_{a z}^{-1}\left(p g_{r}^{r-z}-((p-1) / 2) D_{z s}-g_{z-1}^{0}\right)+\left((p-1)^{2} / 2\right)\left(D_{a s}+E_{a s}\right) \\
\equiv \pi_{a z}^{-1}\left((p-1) g_{r}^{r-z}\right)+((p-1) / 2) B_{a n}^{0}
\end{gathered}
$$

or

$$
\pi_{a z}^{-1}\left(g_{r}^{r-z}\right) \equiv((p-1) / 2) B_{a n}^{0}+((p-1) / 2)\left(D_{a s}+E_{a s}\right)+\pi_{a z}^{-1}\left(g_{z-1}^{0}\right)
$$

since $\pi_{a z}^{-1}\left(D_{z s}\right) \equiv p\left(D_{a s}+E_{a s}\right)$.

Thus there is a divisor equivalent to $\pi_{a z}^{-1}\left(g_{r}^{r-z}\right)$ not invariant under $\langle\psi\rangle$. By Corollary 2.3, the dimension of $\left|\pi_{g z}^{-1}\left(g_{z}^{r-z}\right)\right|$ must be greater than that of $\left|g_{z}^{r-z}\right|$. 


\section{Full ramified cyclic coverings}

Let $W_{z}$ be a Riemann surface, and let $D_{z}\left(=x_{1}+x_{2}+\cdots+x_{s}\right)$ be a divisor on $W_{z}$ of $s$ distinct points $(s \geq 2)$. A cyclic covering of prime degree $p, W_{g}$, ramified over $D_{z}$ is defined by $y^{(1 / p)}$ where $y \varepsilon M\left(W_{z}\right)$ and

$$
(y)=a_{1} x_{1}+a_{2} x_{2}+\cdots+a_{s} x_{s}+p C_{z}
$$

where $0<a_{i}<p, \quad i=1,2, \ldots, s$ and $\sum a_{i}+p\left(\operatorname{deg} C_{z}\right)=0$. We say that $\left\{a_{1}, a_{2}, \ldots a_{s}\right\}$ are the rotation numbers for the covering. Since $y^{(k / p)}, k \neq 0$ $(\bmod p)$ also defines $W_{g}$, the rotation numbers are only defined up to an integral multiple $\bmod p$. The rotation numbers are also defined topologically by a homomorphism from the fundamental group $\pi_{1}\left(W_{z}-D_{z}, \cdot\right)$ onto $Z_{p}$ whose kernel corresponds to the covering. The rotation numbers are the values of the homomorphism on the paths that "circle" the $a_{i}$ 's.

THEOREM 6.1. A ramified cyclic covering of prime degree $p(\geq 2)$ is full if and only if the rotation numbers are all equal to one another.

Proof. Set $s=t p . \quad \operatorname{Deg}\left((p-1) D_{z}+p K_{z}\right)=p((p-1) t+(2 z-2)) \geq p(2 z)$ where $(p-1) t+2 z-2 \geq z$. Consequently, on $W_{z}$ there are $p^{2 z}$ linear series $|G|$ where

$$
p G \equiv(p-1) D_{z}+p K_{z}
$$

If $f \varepsilon M\left(W_{z}\right)$ has the right and left hand sides of (6.1) as zeros and poles then $f^{(1 / p)}$ defines a covering $\pi: W_{g} \rightarrow W_{z}$ where $\left(h=f^{(1 / p)}, \pi\left(D_{g}\right)=D_{z}\right)$

$$
(h)=(p-1) D_{g}+\pi^{-1}\left(K_{z}\right)-\pi^{-1}(G)
$$

Since $K_{g} \equiv(p-1) D_{g}+\pi^{-1}\left(K_{z}\right)$ the covering is full with equal rotation numbers. But there are $p^{2 z}$ cyclic coverings with equal rotation numbers, so they all arise in this manner. Thus equal rotation numbers imply fullness.

For the converse reverse the argument starting with (6.2) multiplied by $p$ and deduce (6.1), that is, that $h^{p} \varepsilon M\left(W_{z}\right)$ and $\left(h^{p}\right)=(p-1) D_{z}+p K_{z}-p G$.

Now we consider half-canonical linear series on $W_{g}$ arising from an equivalence $2 H \equiv G$ for a solution $G$ on $W_{z}$ of $(6.1)$. (Now $p \geq 3$ ) We have $2^{2 z}$ possible $H^{\prime}$ 's with $|H|=g_{(g-1) / p}^{r}$ where $r \geq(g-1) / p-z$.

Let $g_{z-1}^{0}$ be one of the $u_{z}$ half-canonical linear series on $W_{z}$ by $(6.1)$ the equivalence

$$
((p-1) / 2) D_{z}+p g_{z-1}^{0} \equiv p H
$$

has $p^{2 z}$ solutions $|H|$, one for each full cyclic covering $W_{g} \rightarrow W_{z}$ ramified over $D_{z}$ On such a $W_{g}$ we have

$$
((p-1) / 2) D_{g}+\pi^{-1}\left(g_{z-1}^{0}\right) \equiv \pi^{-1}(H)
$$


$\left|\pi^{-1}(H)\right|$ has dimension greater than that of $|H|$ since the divisor on the left hand side of (6.3) is not lifted from $W_{z}$.

We see that the vanishing of the theta function at half periods (corresponding to the existence of half-canonical linear series) for a ramified full $p$ sheeted cyclic covering $(p \geq 3)$ mimics those of full $p$-sheeted dihedral coverings.

Now we wish to show that in moduli space for genus $g$ the full $p$-sheeted cyclic coverings of a $W_{z}$ are in the closure of the space of full $p$-sheeted dihedral coverings of a $W_{z}$, assuming that

$$
2 g-2=p(2 z-2)+s(p-1), \quad p \mid s, \quad s>0 .
$$

On a Riemann surface of genus $z$, pick a divisor $C$ of $s$ distinct points. We want a divisor $D$ of degree $2 s / p$ so that $2 C \equiv p D$. That such a $D$ exists follows if $2 s / p \geq z$. To insure that $C$ and $D$ are disjoint it is useful to have $D$ move in a pencil, so we will assume $2 s / p>z$. Thus we are assuming $4 g-4>$ $p^{2} z+3 p z-4 p$. (If $p=3$ and $z=1$ then $2 g>5$ ). Assuming that $C$ and $D$ are disjoint, let $F \varepsilon M\left(W_{z}\right)$ have divisor: $(F)=p D-2 C$. Now let $y=1-\lambda F$, where the function $y$ depends on the complex parameter $\lambda(\neq 0$.$) Then$

$$
\begin{aligned}
(y)_{0} & =(F)_{(1 / \lambda)}:=B_{\lambda} \\
(y)_{\infty} & =(F)_{\infty}=2 C \\
(y)_{1} & =(F)_{0}=p D
\end{aligned}
$$

Note that $4 /(1-y)=4 /(\lambda F)$.

For all but a finite number of $\lambda$ 's $(\neq 0) B_{\lambda}$ will consist of $2 s$ distinct points so we shall always make this assumption as $\lambda \rightarrow 0$. For a fixed $\lambda \neq 0$ we follow the procedure of section 3 to obtain a full $p$-sheeted dihedral covering, $W_{g}$, of $W_{z}$ with $(p-1) / 2$ ordinary ramification points lying over each of the $2 s$ points of $B_{\lambda}$.

Again let $Y=\sqrt{y}, Z=(1-Y) /(1+Y)$, and $U=Z^{(1 / p)}$. Now let $T=$ $U+U^{-1}$, a function on $W_{g}$ since $T$ is invariant under $\varphi$. T satisfies an equation of the type

$$
T^{p}+Q(T)=Z+Z^{-1}
$$

where $Q$ is a polynomial of degree less than $p$. For example

$$
\begin{array}{ll}
p=3 & T^{p}+Q(T)=T^{3}-3 T \\
p=5 & T^{p}+Q(T)=T^{5}-5 T^{3}+5 T
\end{array}
$$

But $Z+Z^{-1}=-2+4 /(1-y)$. Thus

$$
T^{p}+Q(T)+2=4 /(\lambda F)
$$

where $F$ is independent of $\lambda$. Substituting $\lambda^{-(1 / p)} T$ for $T$ we have

$$
T^{p}+\lambda Q\left(\lambda^{-(1 / p)} T\right)+2 \lambda=4 / F .
$$


Letting $\lambda \rightarrow 0$ this becomes $T^{p}=4 / F$, the equation of a full $p$-sheeted covering of $W_{z}$ with total ramification points over the $s$ distinct points of $C$. (We omit the details of passing to the limit.)

A slightly more complicated argument would deal with the case where $C$ and $D$ are not disjoint.

\section{Full elliptic-trigonal Riemann surfaces}

THEOREM 7.1. Let $W_{g} \rightarrow W_{1}$ be a full three-sheeted covering of Riemann surfaces of genus $g$ and one. $(g=3 r+1, r \geq 3)$. Then $W_{g}$ admits four complete half-canonical linear series $g_{3 r}^{r-1}, h_{3 r}^{r-1}, k_{3 r}^{r-1}, l_{3 r}^{r}$ whose sum is bicanonical (a quartet.) The first three are composite being the lifts of linear series on $W_{1}$, and the fourth is fixed point free and simple being the completion of a lift of a linear series on $W_{1}$. $l_{3 r}^{r}$ is the unique linear series of degree $3 r$ and dimension $r$ or greater.

Proof. Theorem 5.1 and the discussion in Section 6 insures the existence of the four linear series. $l_{3 r}^{r}$, being the lift of a $l_{r}^{r-1}$, is fixed point free, and having more divisors than $l_{r}^{r-1}$, it is simple. If another linear series $G_{3 r}^{r}$ exists then $l_{3 r}^{r}+G_{3 r}^{r}$ has dimension at least $3 r$ (Lemma 2.18) and so is canonical. Since $l_{3 r}^{r}$ is half-canonical, $l_{3 r}^{r}=G_{3 r}^{r}$. The same result for a $G_{3 r}^{r+\varepsilon}(\varepsilon \geq 0)$ now follows.

On $W_{1}$ let $g_{r}^{r-1}, h_{r}^{r-1}, k_{r}^{r-1}, l_{r}^{r-1}$ be the linear series that lift to the four on $W_{g}$. On $W_{1}$ we have

$$
g_{r}^{r-1}+h_{r}^{r-1} \equiv k_{r}^{r-1}+l_{r}^{r-1}
$$

so on $W_{g}$ we have

$$
g_{3 r}^{r-1}+h_{3 r}^{r-1} \equiv k_{3 r}^{r-1}+l_{3 r}^{r} .
$$

Since each linear series is half-canonical, their sum is bi-canonical.

THEOREM 7.2. Let $W_{g}$ be a compact Riemann surface $(g=3 r+1, r \geq 3)$ admitting a quartet $g_{3 r}^{r-1}, h_{3 r}^{r-1}, k_{3 r}^{r-1}, l_{3 r}^{r}$, where $l_{3 r}^{r}$ is the only half-canonical linear series on $W_{g}$ of degree $3 r$ and dimension $r$. Then $W_{g}$ is a full elliptic-trigonal Riemann surface.

Proof. We first show that $l_{3 r}^{r}$ is simple and without fixed points. Suppose $l_{3 r}^{r}$ is composite. By Lemma $2.15 W_{g}$ is not trigonal. Therefore, $W_{g}$ admits a $\gamma_{2}(q)$ where $W_{q}$ admits a complete $l_{(3 r-f) / 2}^{r}$ (where $l_{3 r}^{r}$ has $f$ fixed points), and $l_{(3 r-f) / 2}^{r}$ is not special (Cliff.) Then $q=(r-f) / 2(\mathrm{RR})$ by Lemma $2.12 W_{g}$ admits many half-canonical $G_{3 r}^{r}$ 's. This contradiction insures that $l_{3 r}^{r}$ is simple. That $l_{3 r}^{r}$ is without fixed points follows from the fact that $2\left(l_{3 r-1}^{r}\right)=g_{6 r-2}^{3 r-1+\varepsilon}, \varepsilon \geq 0$ (Lemma 2.16) and that $W_{g}$ is not hyperelliptic (Cliff.)

By Lemma 2.7 there exists an unramified 4-sheeted Galois covering $W_{4 g-3} \rightarrow W_{g}$ and $W_{4 g-3}$ admits a simple $g_{12 r}^{4 r}(12 r \geq 36$.) By a theorem of Eisenbud-Harris [6, p 102] $W_{4 g-3}$ is trigonal, elliptic-trigonal, or admits a $g_{n}^{1}$ 
imposing two conditions on $g_{12 r}^{4 r}$. By Lemma $2.17 n=4$. It follows that $W_{g}$ admits one of the three mutually exclusive alternatives.

Assuming that $W_{12 r+1}$ is elliptic-trigonal, it follows by Lemma 2.8 (applied twice) that $W_{3 r+1}$ is also. Fullness will follow from the following lemma.

Lemma 7.3. Let $W_{3 r+1}(r \geq 3)$ admit a simple half-canonical $l_{3 r}^{r}$ and an elliptic-trigonal covering $\pi: W_{3 r+1} \rightarrow W_{1}$. If a divisor $D$ of $l_{3 r}^{r}$ contains two points of a fiber of $\pi$, then $D$ is the lift of a divisor of degree $r$ on $W_{1}$. Thus $\pi$ is a full covering.

Proof of Lemma 7.3. Let $E$ be a divisor in $\gamma_{3}(1)$, two points of which are in $D$. Let $F$ be another divisor in $\gamma_{3}(1)$ which has a point in $D$. Then $|E+F|$ is a $g_{6}^{1}$ on $W_{g}$ (being the lift of a $g_{2}^{1}$ ) and so imposes three conditions on $l_{3 r}^{r}$ (Lemma 2.9.) Thus $(E+F, D)=E+F$. Now $|D|=l_{3 r}^{r}, D-(E+F)=l_{3 r-6}^{r-3}$, and so $|D-E|=l_{3 r-3}^{r-2}$. Consequently, $l_{3 r-3}^{r-2}-F=l_{3 r-6}^{r-3}$. Thus $F$ imposes one condition on $l_{3 r-3}^{r-2}, l_{3 r-3}^{r-2}$ is the lift of a $g_{r-1}^{r-2}$ on $W_{1}$, and the result follows.

Since $W_{g}$ is not trigonal, to complete the proof we must show that $W_{g}$ does not admit a $g_{4}^{1}$. Suppose that $W_{g}$ does admit a $g_{4}^{1}$. We will arrive at a contradiction.

We first show that if $r \geq 5$ then $l_{3 r}^{r}-g_{4}^{1}\left(=l_{3 r-4}^{r-2}\right)$ is simple and without fixed points. Remember that $g_{4}^{1}$ imposes at most two conditions on $l_{3 r}^{r}$, and therefore, at most two conditions on any subseries of dimension at least 2 . If $l_{3 r-4}^{r-2}$ is composite and compounded of $g_{4}^{1}$ then $l_{3 r-4}^{r-2} \equiv(r-2) g_{4}^{1}+D_{f}$, and so $3 r-4 \geq$ $4 r-8$, a contradiction. If $l_{3 r-4}^{r-2}$ is compounded of a $\gamma_{t}(q)$ then $t$ divides 4 , and so $t=2$. As earlier in this proof, $W_{q}$ admits a non-special $g_{(3 r-4-f) / 2}^{r-2}$ and $q=(r-f) / 2$. Again this leads to too many half-canonical $G_{3 r}^{r}$ 's. If $l_{3 r-4}^{r-2}$ has a fixed point then so does $l_{3 r}^{r}\left(=l_{r}^{r-2}+g_{4}^{1}\right)$, a contradiction.

We now proceed for all $r \geq 3$. For $r=4$ we assume that $l_{12}^{4}-g_{4}^{1}\left(=l_{8}^{2}\right)$ is simple. (Note that the preceding argument shows that if $l_{8}^{2}$ is composite then the only possibility is $\left|2 g_{4}^{1}\right|=l_{8}^{2}$.) If $r=3$ we assume that $l_{9}^{3}-g_{4}^{1}\left(=l_{5}^{1}\right)$ is without fixed points. (The only other possibility is that $l_{5}^{1}=g_{4}^{1}+x$, where $x$ is a fixed point.)

By Lemma $2.10 l_{3 r-4}^{r-2}$ imposes at most $r-1$ conditions on the other linear series in the quartet, and if $r \geq 4$ they are all simple as a consequence. (The author is indebted to L. Donohoe [5] for the basic idea in the following discussion as well as many of the details.)

Suppose that $g_{3 r}^{r-1} \equiv l_{3 r-4}^{r-2}+S$, where $S$, a divisor of degree 4 , is not in $g_{4}^{1}$. By doubling this last equivalence, it follows that $|2 S| \equiv 2 g_{4}^{1}$, and Lemma 2.14 shows that $S=P+Q$ where $|2 P| \equiv|2 Q| \equiv g_{4}^{1}$, and $(P, Q)=0$. Then

$$
g_{3 r}^{r-1}-P \equiv l_{3 r-4}^{r-2}+Q \equiv l_{3 r}^{r}-Q
$$

$Q$ imposes one or two conditions on $l_{3 r}^{r}$ since $l_{3 r}^{r}$ is without fixed points. If $Q$ imposes one condition on $l_{3 r}^{r}$ then $P$ is a fixed divisor for $g_{3 r}^{r-1}$. 
Suppose $Q$ imposes two conditions on $l_{3 r}^{r}$. Then $l_{3 r}^{r}-Q:=l_{3 r-2}^{r-2}$. By BN $l_{3 r}^{r}+Q$ has dimension $r$. Since $g_{3 r}^{r-1} \equiv l_{3 r-4}^{r-2}+P+Q$, and $g_{3 r}^{r-1}+P \equiv l_{3 r}^{r}+Q$, we see that $g_{3 r}^{r-1}$ has $Q$ as a fixed divisor and $g_{3 r}^{r-1}-Q \equiv l_{3 r-4}^{r-2}+P$.

In all cases $g_{3 r}^{r-1}$ has a divisor of fixed points of degree two, $P_{g}$, and there is another divisor $Q_{g}\left(2 P_{g} \equiv 2 Q_{g} \equiv g_{4}^{1}\right)$ so that

$$
g_{3 r}^{r-1}-P_{g} \equiv l_{3 r-4}^{r-2}+Q_{g}\left(:=g_{3 r-2}^{r-1}\right)
$$

By the same argument there exist $P_{h}, Q_{h}$ (resp $P_{k}, Q_{k}$ ), divisors of degree two, so that $P_{h}\left(\operatorname{resp} P_{k}\right)$ is a divisor of fixed points for $h_{3 r}^{r-1}\left(\operatorname{resp~} k_{3 r}^{r-1}\right)$ and

$$
\begin{aligned}
& h_{3 r}^{r-1}-P_{h} \equiv l_{3 r-4}^{r-2}+Q_{h}\left(:=h_{3 r-2}^{r-1}\right) \\
& k_{3 r}^{r-1}-P_{k} \equiv l_{3 r-4}^{r-2}+Q_{k} \quad\left(:=k_{3 r-2}^{r-1}\right)
\end{aligned}
$$

We claim that $g_{3 r-2}^{r-1}=h_{3 r-2}^{r-1}=k_{3 r-2}^{r-1}$. For if the first two are not equal then $g_{3 r-2}^{r-1}+h_{3 r-2}^{r-1} \equiv g_{6 r-4}^{3 r-3+\varepsilon}$ which is special (Lemma 2.18, RR.) By Cliff and BN we see that $\varepsilon=0$, and $g_{3 r-2}^{r-1}+h_{3 r-2}^{r-1}+g_{4}^{1} \equiv K_{g}$. By replacing $g_{4}^{1}$ by $2 P_{g}$ and noting that $g_{3 r-2}^{r-1}+P_{g}$ is half canonical we see that $g_{3 r-2}^{r-1}=h_{3 r-2}^{r-1}$ after all. Call this linear series $m_{3 r-2}^{r-1}$. Then we have:

$$
\begin{aligned}
m_{3 r-2}^{r-1} & =g_{3 r}^{r-1}-P_{g} \equiv l_{3 r-4}^{r-2}+Q_{g} \\
& =h_{3 r}^{r-1}-P_{h} \equiv l_{3 r-4}^{r-2}+Q_{h} \\
& =k_{3 r}^{r-1}-P_{k} \equiv l_{3 r-4}^{r-2}+Q_{k}
\end{aligned}
$$

Consequently $Q_{g}=Q_{h}=Q_{k}(:=Q)$ and $l_{3 r}^{r} \equiv m_{3 r-2}^{r-1}+Q$. Thus $2 P_{g} \equiv 2 P_{h} \equiv$ $2 P_{k} \equiv 2 Q$ and $P_{g}+P_{h} \equiv P_{k}+Q$. By Lemma 2.7 it follows that there is a smooth Galois covering $W_{4 g-3} \rightarrow W_{g}$ and $W_{4 g-3}$ admits a $g_{8}^{3}$ which is necessarily composite. Thus $W_{4 g-3}$ is elliptic-hyperelliptic and $W_{g}$ is $q$-hyperelliptic $(q=0$ or 1.) We have reached the desired contradiction.

Now we must consider the exceptional cases.

For $r=4$ assume $l_{12}^{4} \equiv 3 g_{4}^{1}$. First we show that $g_{12}^{3}$ is simple. If not then $W_{13}$ admits a $\gamma_{t}(q)$ where $t$ divides 4 ; thus $t=2$. $\quad W_{q}$ admits a $g_{6-f}^{3}(f$ fixed points for $\left.g_{12}^{3}\right)$ and $W_{q}$ is hyperelliptic. Since $l_{12}^{4}$ is simple, Lemma 2.11 implies that $q \geq 4$. But then $f=0$, and $g_{6}^{3} \equiv 3 g_{2}^{1}$, and this gives $g_{12}^{3} \equiv 3 g_{4}^{1}$, a contradiction. Thus $g_{12}^{3}$ is simple.

If $g_{12}^{3}$ has one fixed point, $g_{12}^{3}=g_{11}^{3}+x$, then by Lemma $2.10 l_{12}^{4}-g_{11}^{3}=y$ and $2 x \equiv 2 y$, a contradiction. If $g_{12}^{3}$ has two fixed points then $g_{12}^{3}=g_{10}^{3}+P$, $l_{12}^{4}-g_{10}^{3}=Q, 2 P \equiv 2 Q \equiv g_{4}^{1}$, and so

$$
g_{12}^{3}-P \equiv l_{12}^{4}-Q
$$

If $g_{12}^{3}$ has 3 fixed points then $W_{13}$ admits a simple $g_{9}^{3}$. Since this implies that $g \leq 12$ we have a contradiction. If $g_{12}^{3}$ has no fixed points then $g_{12}^{3}-g_{4}^{1}=g_{8}^{1}$. $l_{12}^{4}-g_{8}^{1}=l_{4}^{0} \quad\left(\right.$ Lemma 2.9), and $2 l_{4}^{0} \equiv 2 g_{4}^{1}$. Thus $l_{4}^{0}=P+Q, 2 P \equiv 2 Q \equiv g_{4}^{1}$, $g_{12}^{3} \equiv g_{8}^{1}+2 P, l_{12}^{4} \equiv g_{8}^{1}+P+Q$, or 


$$
g_{12}^{3}-P \equiv l_{12}^{4}-Q .
$$

Exactly the same argument can be applied to $h_{12}^{3}$ and $k_{12}^{3}$ and we get equivalences analogous to (7.2) and (7.3). Then we can apply the argument following (7.1) and reach the conclusion that $W_{13}$ does not admit a $g_{4}^{1}$.

The case $r=3$ starts with $l_{9}^{3} \equiv 2 g_{4}^{1}+x=g_{4}^{1}+l_{5}^{1}$ where $l_{5}^{1}=g_{4}^{1}+x$. We first show that $g_{9}^{2}$ is simple. If $g_{9}^{2}$ is composite and $W_{10}$ admits a $g_{4}^{1}$ (which is unique) then $W_{10}$ admits a $\gamma_{2}(q)$ where $W_{q}$ admits a $g_{4}^{2}$ and is hyperelliptic and by Lemma $2.11 q \geq 3$. Thus $g_{4}^{2}=2 g_{2}^{1}, g_{9}^{2} \equiv 2 g_{4}^{1}+y$, and $2 x \equiv 2 y$. This contradiction shows that $g_{9}^{2}$ is simple.

If $g_{9}^{2}$ is simple it cannot admit only one fixed point $\left(g_{9}^{2}=g_{8}^{2}+x, l_{9}^{3} \equiv g_{8}^{2}+y\right.$, $2 x \equiv 2 y$, contradiction, as before.) It cannot admit three fixed points $\left(l_{9}^{3}-g_{6}^{2} \equiv\right.$ $g_{3}^{1}$, Lemma 2.10.) If $g_{9}^{2}$ admits a fixed divisor $P$ of degree 2 , then $g_{9}^{2}=g_{7}^{2}+P$, $l_{9}^{3} \equiv g_{7}^{2}+Q, 2 P \equiv 2 Q$, and we have

$$
l_{9}^{3}-Q \equiv g_{9}^{2}-P
$$

If $g_{9}^{2}$ is without fixed points then $g_{9}^{2} \equiv g_{4}^{1}+g_{5}^{0} . \quad 2 g_{5}^{0} \equiv 2 l_{5}^{1} \equiv 2 g_{4}^{1}+2 x \equiv l_{9}^{3}+x$. $2 g_{4}^{1}\left(\equiv g_{8}^{2}\right) \equiv K_{10}-2 l_{5}^{1}$. By Cliff $2 l_{5}^{1}\left(\equiv 2 g_{5}^{0} \equiv l_{10}^{3}\right)$ has dimension 3 and so has $x$ for a fixed point. Consequently, $x \varepsilon g_{5}^{0}, g_{5}^{0}=g_{4}^{0}+x, 2 g_{4}^{0}+2 x \equiv 2 l_{5}^{1} \equiv 2 g_{4}^{1}+2 x$, or $2 g_{4}^{1} \equiv 2 g_{4}^{0} . \quad g_{4}^{0} \equiv P+Q, 2 P \equiv 2 Q,(P, Q)=0$, and we have

$$
l_{9}^{3}-Q \equiv g_{9}^{2}-P
$$

For each of the linear series $g_{9}^{2}, h_{9}^{2}, k_{9}^{2}$ we have formulas like (7.4) and (7.5), and we conclude the proof for the case $g=10$ as in the proof following formula (7.1). For $r \geq 3$ the proof that $W_{3 r+1}$ does not admit a $g_{4}^{1}$ is complete.

The case $g=7$ was considered in [4].

By CS it follows that only for $g \leq 10$ can a $W_{g}$ admit several elliptictrigonal coverings. If $W_{10}$ covers a torus in three sheets then by Lemma 2.4 there can be only one quartet lifted from this torus to $W_{10}$. Thus there is a oneto-one correspondence between full three-sheeted coverings $W_{10} \rightarrow W_{1}$ and quartets on $W_{10}$.

\section{REFERENCES}

[1] Accola, R. D. M., Riemann surfaces, theta functions and abelian automorphism groups, Lecture Notes in Mathematics, no. 483, Springer, 1975.

[2] Accola, R. D. M., On Castelnuovo's inequality for algebraic curves, I, Trans of the AMS 257 (1979), 357-373.

[ 3 ] Accola, R. D. M., Plane models for Riemann surfaces admitting certain half-canonical linear series, Part II, Trans of the AMS 263 (1981), 243-259.

[4] Accola, R. D. M., Some loci in Teichmuller space for genus seven defined by vanishing thetanulls, Manuscripta Mathematica 81 (1993), 113-127.

[ 5 ] Donohoe, L., Plane models for Riemann surfaces admitting half-canonical $g_{9}^{2}$ 's and $g_{9}^{3}$ 's. 
[6] Harris, J., Curves in projective space, L'universite de Montreal (1982).

[7] KanI, E., Relations between the genus and between the Hasse-Witt invariants of Galois coverings of curves, Canadian_Math. Bull 28(3) (1985), 321-327.

[ 8 ] Lewittes, J., Riemann surfaces and the theta function, Acta Mathematica 111 (1964), 37-61.

Department of Mathematics

BROWN UNIVERSITY

Providence, RI, 02912

E-mail: raccola@math.brown.edu 